Ophthalmologe 2015 · 112:870

DOI 10.1007/s00347-015-0145-0

Online publiziert: 5. Oktober 2015

(c) Springer-Verlag Berlin Heidelberg 2015

CrossMark

Deutsche Ophthalmologische Gesellschaft

München, Deutschland

\title{
Erratum zu: Abstract-Band DOG 2015
}

\section{Erratum zu:}

Ophthalmologe 2015 - [Suppl 1]: 112:S1-S179

DOI 10.1007/s00347-015-0116-5

Das Abstract PDO-03-04 „Untersuchung der okuläre Biomechanik normaler und keratokonischer Augen (mit/ohne Crosslinking) mittels dynamischer Ultra-HighSpeed-Schleimpflug-Messung “ enthielt Fehler.

Bitte beachten Sie die korrigierte Version des Abstracts.

Untersuchung der okuläre Biomechanik normaler und keratokonischer Augen (mit/ohne Crosslinking) mittels dynamischer Ultra-High-SpeedSchleimpflug-Messung

Fuchsluger T. ${ }^{1}$, Brettl S. ${ }^{2}$, Geerling G. ${ }^{3}$, Franko Zeitz $P^{4}$

${ }^{1}$ Universitätsklinik Erlangen, Augenheilkunde, Erlangen, Deutschland,

${ }^{2}$ Heinrich-Heine-Universität, Düsseldorf, Deutschland,

${ }^{3}$ Universitätsaugenklinik, Düsseldorf,

Deutschland,

${ }^{4}$ Düsseldorf, Deutschland

Hintergrund. Vergleich neuer okulärer biomechanischer Parameter der Deformation von Hornhäuten normaler und keratokonischer Augen (mit oder ohne Crosslinking, CXL), gemessen mittels Hochgeschwindigkeits-ScheimpflugTechnologie (Corvis ST, Oculus Optikgeräte $\mathrm{GmbH}$, Wetzlar).

Methodik. Vergleichende Studie von 67 Augen mit Keratokonus (Gruppe 1: 39 Augen ohne Crosslinking, Gruppe 2: 28 Augen nach Crosslinking) und 50 Augen Kontrolle (Gruppe 3: kein Keratokonus).

Die Online-Version des Originalartikels ist unter doi:10.1007/s00347-015-0116-5 zu finden.
Neben Refraktion und Visus wurden jeweils die Applanationsparameter Länge "length“ (in mm) und Geschwindigkeit "velocity“ (in $\mathrm{m} / \mathrm{s}$ ) bei den messungsinhärenten zwei Applanationen analysiert. Weiterhin erfolgte die Messung der Pachymetrie, des Krümmungsradius am Deformationsendpunkt (in $\mathrm{mm}$ ) und der Deformationsamplitude (in mm). Diese Messerwerte wurden um Daten der Pentacam erweitert: (Pachymetrie (dünnste Stelle (in $\mu \mathrm{m})$ ), K1 (in dpt), K2 (in dpt), Achse (in ${ }^{\circ}$ ), Kmax (in dpt) und Posterior float (in $\mu \mathrm{m})$ ).

Ergebnisse. Die Applanationslängen waren bei Keratokonusaugen signifikant niedriger als bei Kontrollaugen (Gr. 1: $1,65 \pm 0,24 \mathrm{~mm}$ und $1,63 \pm 0,47 \mathrm{~mm}$; Gr. 2: 1,62 $\pm 0,27 \mathrm{~mm}$ und $1,27 \pm 0,40 \mathrm{~mm}$; Gr. 3: $1,74 \pm 0,12 \mathrm{~mm}$ und $1,74 \pm 0,24 \mathrm{~mm}$, mit Ausnahme von Applanation 2 bei Konus vs Kontrolle. Die zweite Applanationsgeschwindigkeit war bei normalen Augen deutlich höher als bei Keratokonus (mit und ohne CXL): Gr. 1: 0,16 $\pm 0,03 \mathrm{~m} / \mathrm{s}$ und $-0,41 \pm 0,09 \mathrm{~m} / \mathrm{s}$ (sign.); Gr. 2 : $0,16 \pm 0,03 \mathrm{~m} / \mathrm{s}$ und $-0,51 \pm 0,09 \mathrm{~m} / \mathrm{s}$ (sign.); Gr. 3: $0,15 \pm 0,02 \mathrm{~m} / \mathrm{s}$ und $-0,37 \pm 0,06 \mathrm{~m} / \mathrm{s}$ (sign.)). Die Unterschiede der Deformationsamplituden im Vergleich der drei Gruppen untereinander waren jeweils signifikant.

Schlussfolgerungen. Die Messung der Hornhautdeformationen mittels UltraHigh-Speed-Scheimpflug liefert wertvolle Informationen über die korneale Biomechanik. Viele Parameter zeigten signifikante Unterschiede im Vergleich der drei Gruppen (Keratokonus unbehandelt versus Keratokonus mit Crosslinking versus normaler Augen). Die Messwerte selbst waren reproduzierbar. Ausführlichere Studien sind nötig für breitere Anwendungen dieser neuen Technologie in der Klinik.

\section{Korrespondenzadresse}

Deutsche Ophthalmologische Gesellschaft

Platenstr.1, 80336 München

geschaeftsstelle@dog.org 